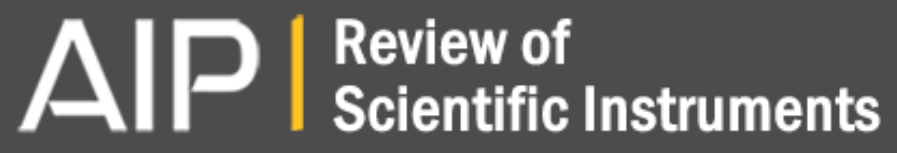

\section{Nested Helmholtz coil design for producing homogeneous transient rotating magnetic fields}

George Podaru, John Moore, Raj Kumar Dani, Punit Prakash, and Viktor Chikan

Citation: Review of Scientific Instruments 86, 034701 (2015); doi: 10.1063/1.4908173

View online: http://dx.doi.org/10.1063/1.4908173

View Table of Contents: http://scitation.aip.org/content/aip/journal/rsi/86/3?ver=pdfcov

Published by the AIP Publishing

\section{Articles you may be interested in}

Pulsed-coil magnet systems for applying uniform 10-30 T fields to centimeter-scale targets on Sandia's Z facility

Rev. Sci. Instrum. 85, 124701 (2014); 10.1063/1.4902566

Magnetic field uniformity of the practical tri-axial Helmholtz coils systems

Rev. Sci. Instrum. 85, 055115 (2014); 10.1063/1.4876480

Planar coil system consisting of three coil pairs for producing a uniform magnetic field

J. Appl. Phys. 99, 08D904 (2006); 10.1063/1.2165107

Compact low field magnetic resonance imaging magnet: Design and optimization

Rev. Sci. Instrum. 71, 1534 (2000); 10.1063/1.1150491

A rotating coil probe for the magnetic field measurement on a long pulsed tokamak

Rev. Sci. Instrum. 70, 445 (1999); 10.1063/1.1149281

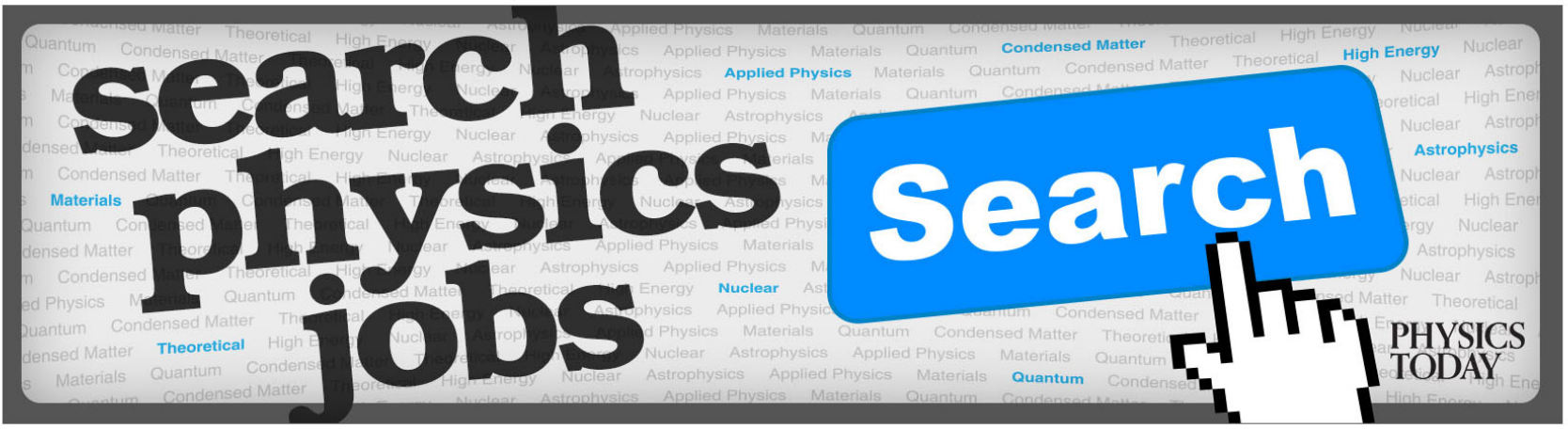




\title{
Nested Helmholtz coil design for producing homogeneous transient rotating magnetic fields
}

\author{
George Podaru, ${ }^{1}$ John Moore, ${ }^{1}$ Raj Kumar Dani, ${ }^{1}$ Punit Prakash, ${ }^{2}$ and Viktor Chikan ${ }^{1, a)}$ \\ ${ }^{1}$ Department of Chemistry, Kansas State University, 213 CBC Building, Manhattan, Kansas 66506-0401, USA \\ ${ }^{2}$ Department of Electrical and Computer Engineering, Kansas State University, Manhattan, \\ Kansas 66506, USA
}

(Received 6 November 2014; accepted 3 February 2015; published online 3 March 2015)

\begin{abstract}
Electromagnets that can produce strong rotating magnetic fields at $\mathrm{kHz}$ frequencies are potentially very useful to exert rotating force on magnetic nanoparticles as small as few nanometers in size. In this article, the construction of a pulsed high-voltage rotating electromagnet is demonstrated based on a nested Helmholtz coil design. The energy for the coils is provided by two high-voltage discharge capacitors. The triggered spark gaps used in the experiments show sufficient accuracy to achieve the high frequency rotating magnetic field. The measured strength of the rotating magnetic field is 200 $\mathrm{mT}$. This magnetic field is scalable by increasing the number of turns on the coils, by reducing the dimensions of the coils and by increasing the discharge current/voltage of the capacitors. (C) 2015 AIP Publishing LLC. [http://dx.doi.org/10.1063/1.4908173]
\end{abstract}

\section{INTRODUCTION}

Rapidly changing magnetic fields are utilized in many areas of science and engineering. In the field of life science, one important area where magnetic fields are utilized is the field of magnetic hyperthermia, ${ }^{1}$ where the alternating or rotating magnetic fields are utilized to produce heat in the sample via Neel or Brownian relaxation of magnetic nanoparticles. $^{2}$ Generally, magnetic nanoparticles can produce useful heat on the order of a few $100 \mathrm{~W} / \mathrm{g} .{ }^{1,3,4}$ Recently, Lee et al. ${ }^{5}$ have shown that if the exchange interaction of the magnetic nanoparticles is used, the number can reach $1000 \mathrm{~W} / \mathrm{g}$ quantities. Recently, Sharapova et al. have shown that by using rotating magnetic fields instead of alternating magnetic fields, the heating efficiency of magnetic nanoparticles has increased significantly. ${ }^{6,7}$ In traditional magnetic hyperthermia, the magnetic field used is a low amplitude sinusoidal magnetic field. ${ }^{1}$ In general, the produced heat is capable of destroying cancer tissue or releasing drug molecules from liposomes ${ }^{8}$ for targeted delivery. However, there are several challenges of this technology so that it reaches its full potential. The required nanoparticle concentration for effective elimination of cancer tissue needs to be on the order of several $\mathrm{mg} / \mathrm{ml}$ to reach needed $42-45^{\circ} \mathrm{C}$ temperature. ${ }^{9-11}$ In addition, magnetic hyperthermia treatments last for several minutes and hours. A more effective way to destroy cancer cells is to utilize the nanoparticles as little magnetically driven drill bits. The mechanical force ${ }^{12}$ can potentially be more effective in eliminating cancer cells if the lipid bilayer can be punctured. ${ }^{13}$ Recent results have shown ${ }^{14}$ that cancer cells are more deformable than healthy cells, which could be utilized in selective cancer cell destruction if combined with mechanical force from rotating or twisting magnetic nanostructures as a result of external magnetic stimuli. In order to rotate magnetic nanoparticles on the order of 10 to

\footnotetext{
a) Author to whom correspondence should be addressed. Electronic mail: vchikan@ksu.edu
}

few tens of nanometer size scale, the magnetic field needs to be increased to overcome the thermal motion of the particles. The required rotating magnetic field strength to manipulate magnetic nanoparticles on the $10-30 \mathrm{~nm}$ diameter range is on the order of a few hundred millitesla. A simple way to generate rotating magnetic fields is via a pair of Helmholtz coils arranged perpendicularly. These designs are widespread and can produce magnetic fields that are few millitesla. Increasing the magnetic field beyond this point is challenging because the continuous current can result resistive heating which requires significant amount of cooling. Cooling of the coils can be facilitated at larger facilities such as the National High Magnetic Field facility. However, the laboratory use of these strong rotating magnets without cooling is desirable for life science based research. In this article, we describe a low duty cycle nested Helmholtz coil system, which is capable of producing strong rotating magnetic pulses without the need of significant cooling.

\section{CONSTRUCTION OF NESTED HELMHOLTZ COILS}

Traditionally, Helmholtz coils are used to generate uniform homogeneous static and alternating magnetic fields in a relatively large volume. The Helmholtz coil design is also used to produce homogeneous rotating magnetic fields when two or three coils are nested within each other. Manipulating the currents inside these coils allows the production of static and rotating magnetic fields in the three dimensional space. Unfortunately, the traditional wire coiling used in Helmholtz coils has its limitation in increasing the strength of the magnetic fields. There are several design challenges of producing strong rotating magnetic fields in a relatively large volume. Early on, Bitter ${ }^{15}$ developed a design based on stacking of concentric discs that minimizes the forces acting on the coil. The current density in traditional solenoid type coils is constant, while in the Bitter coil designs, the current density falls with $1 / \mathrm{r}$ producing a more favorable condition for high magnetic fields. 


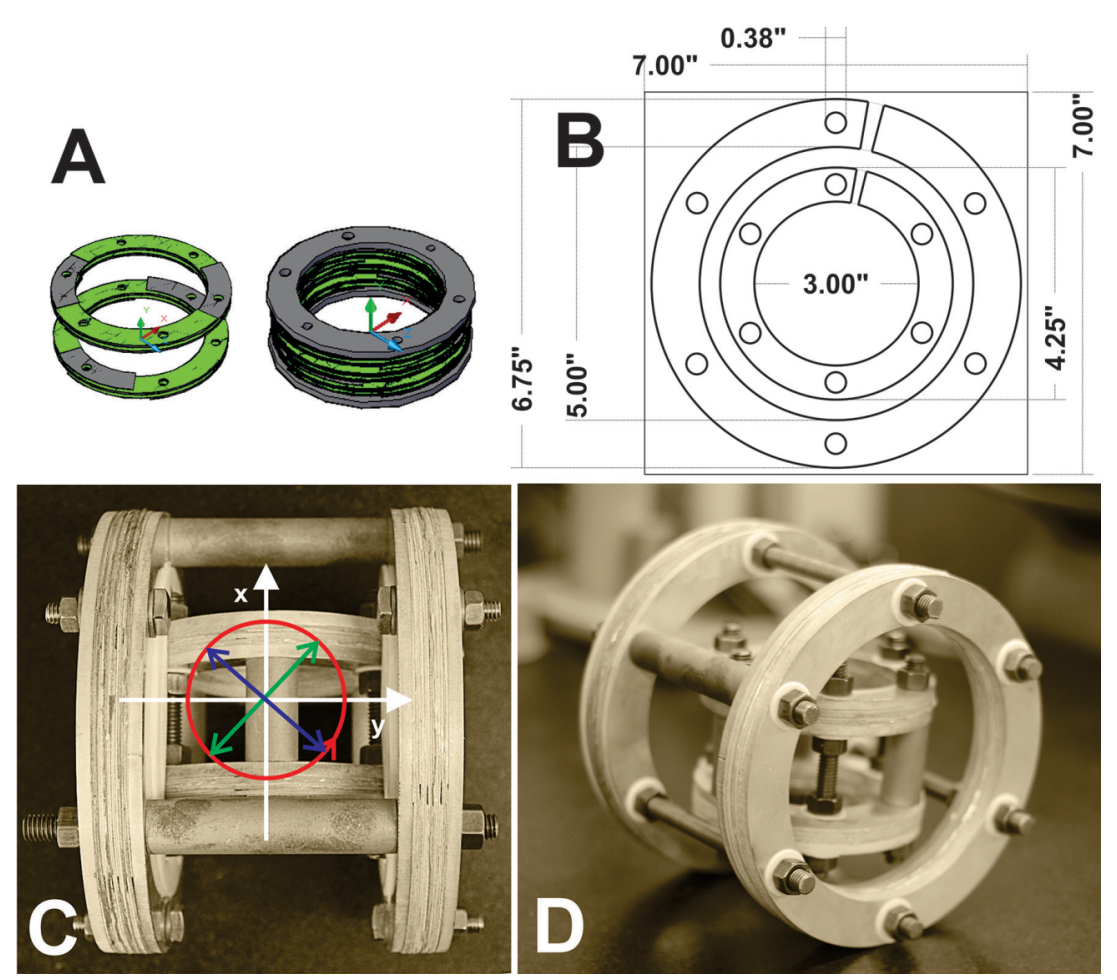

FIG. 1. (a) Assembly of the bitter disks. (b) Dimensions of the bitter disk used to produce the nested Helmholtz coil. (c) Top view of the Helmholtz coil indicating the directions of the magnetic field produced in this paper. (d) Perspective picture of the nested Helmholtz coil without the Teflon insert.

The additional advantage of the bitter coil design is that it distributes the mechanical stress from the Lorentzian force more evenly than in the wire coiled solenoid and also allows efficient cooling if holes for liquid coolant are introduced in the bitter disks. In our research, we combine the Bitter design of electromagnet (Figure 1) to form a 2D Helmholtz coil system for producing rotating magnetic fields. The Bitter disks are manufactured from copper-beryllium alloy to increase the tensile strength of the coil material to resist the mechanical stress from the Lorentzian forces. The tensile strength of copper is $220 \mathrm{MPa}$ while the copper beryllium used in this work has a tensile strength of $820 \mathrm{MPa}$. The conductivity of copper beryllium ( $1 / 2$ HT tempered C17410 alloy) is $50 \%$ of the conductivity of copper. The Bitter disks are electrochemically plated with silver, which further increases the conductivity of the coils and reduces the contact resistance. The bitter disks are separated by mica sheets and sandwiched between the silver coated copper end plates. The packing order and the assembly of the bitter disks are also shown in Figure 1. The two parts of a single Helmholtz coil are held together by brass rods that are insulated from the electrical components of the coils. The spacers between the two parts of the coil are manufactured from high conductivity copper rods. These rods are also silver plated for optimal conductivity. The smaller nested Helmholtz coil is assembled with the help of a Teflon insert, which secures the smaller coil inside the larger coil. The Teflon insert can be rotated with the inner coil so that the main rotating axis of the rotating magnet can be changed.

The nested Helmholtz coils are calibrated with the help of $60 \mathrm{~Hz}$ alternating current from the electrical outlet. 12A RMS current is passed through both of the coils, and the magnetic fields of the two coils are measured with a low frequency
AC Gauss meter. The output from the Gauss meter has been recorded and shown in Figure 3 below. These results show that in order to achieve the same magnitude magnetic fields at the center of the two coils for the circular polarized fields, approximately twice as much current has to pass through in the larger coil than in the smaller coil. In these experiments, the current in the larger coil is increased by using higher discharge voltage in that coil. By careful choice of the number of disks used in each coil, the same amount of magnetic fields can be achieved with the same amount of current. The data in Figure 3 also allow calibrating the Rogowski coils used in the high voltage experiments.

The rotating magnetic field is produced by discharging high voltage capacitors via the coils of the Helmholtz magnet. The circuit design is shown in Figure 2 for a single Helmholtz coil. Two identical circuits are used to control the magnetic field independently from each of the nested Helmholtz coils. One of them is shown in Figure 2. The capacitor is discharged with the help of a homemade triggered spark gap as shown in Figure 2. The trigger pulse is a high frequency $0.5 \mathrm{~J} 20 \mathrm{kV}$ pulse with a duration of $500 \mathrm{~ns}$ that initiates the trigger after charging the main capacitor. The high frequency current is monitored by a homemade Rogowksi coil, which produces voltages proportional to the $\mathrm{dI} / \mathrm{dt}$ in each coil. The voltages are measured by attenuated probes and recorded by an oscilloscope. The signal is then integrated to produce the current response of the coils. The two independent discharge circuits for each of the Helmholtz coils are connected via a digital transistor-transistor logic (TTL) timing box, which allows precise control of the magnetic pulses with respect to each other. The TTL pulses are used for timing signal to trigger the spark gaps of each coil independently. 

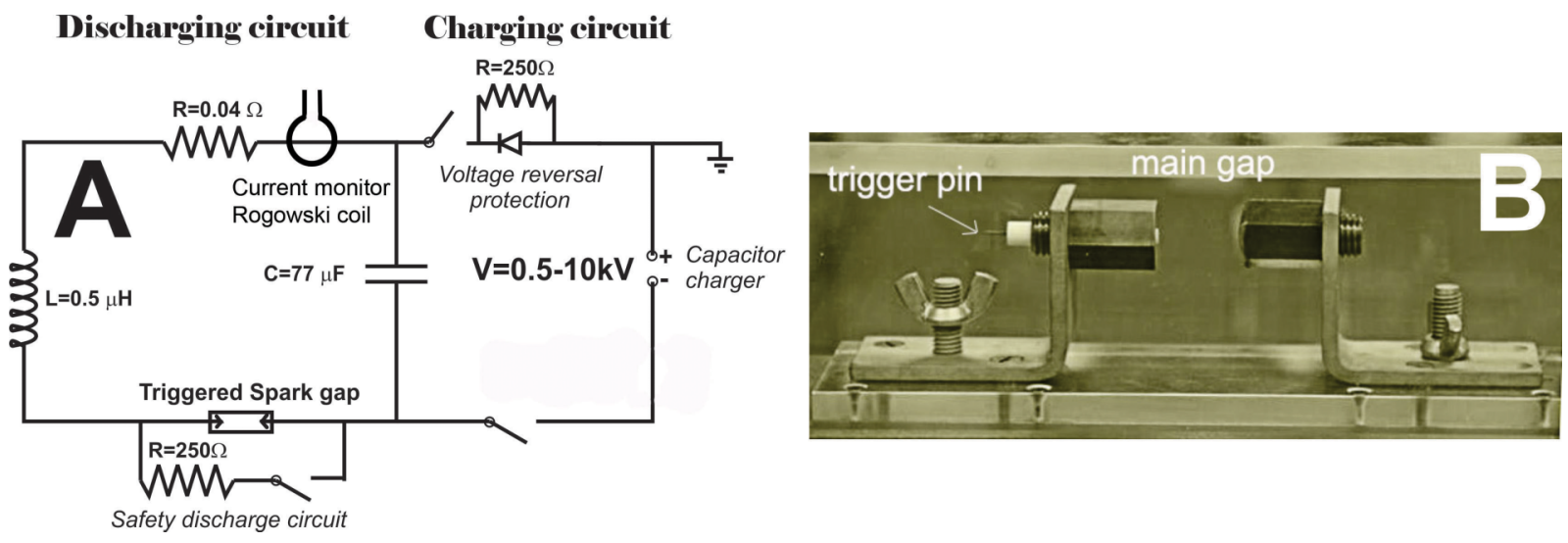

FIG. 2. (a) Schematics of charging and discharging circuit of a single coil for nested Helmholtz coil design. (b) Picture of the home made spark gap with adjustable gap.

The magnitude of the magnetic field applicator is also measured via the Faraday rotation of an optical material, such as water or borosilicate glass, with known optical constant (Figure 5). ${ }^{16}$ This procedure is necessary to ensure that the electrical sensor properly functions at both low and high magnetic fields. The optical measurement of the magnetic field of the coils is as follows: The strong magnetic field induces birefringence of an optical material. The induced birefringence of the material rotates the plane of polarization of a linearly polarized light. This rotation angle $(\theta)$ varies according to Faraday equation: $\theta=v B 1$, where $v$ is Verdet constant of the material, $\mathrm{B}$ is the magnetic field, and $\mathrm{l}$ is the optical path length. The magnitude of the Faraday rotation of optical materials is linearly proportional to the amount of magnetic field, which is utilized in the calibration of pulsed magnetic fields for the experiments described in this paper. For the calibration of magnetic fields, water is used with known optical pathlength $(1 \mathrm{~cm})$. The Faraday rotation constant (Verdet constant) of these materials is published in the literature. ${ }^{17} \mathrm{~A}$ linearly polarized $632 \mathrm{~nm}$ HeNe laser is passed through the water

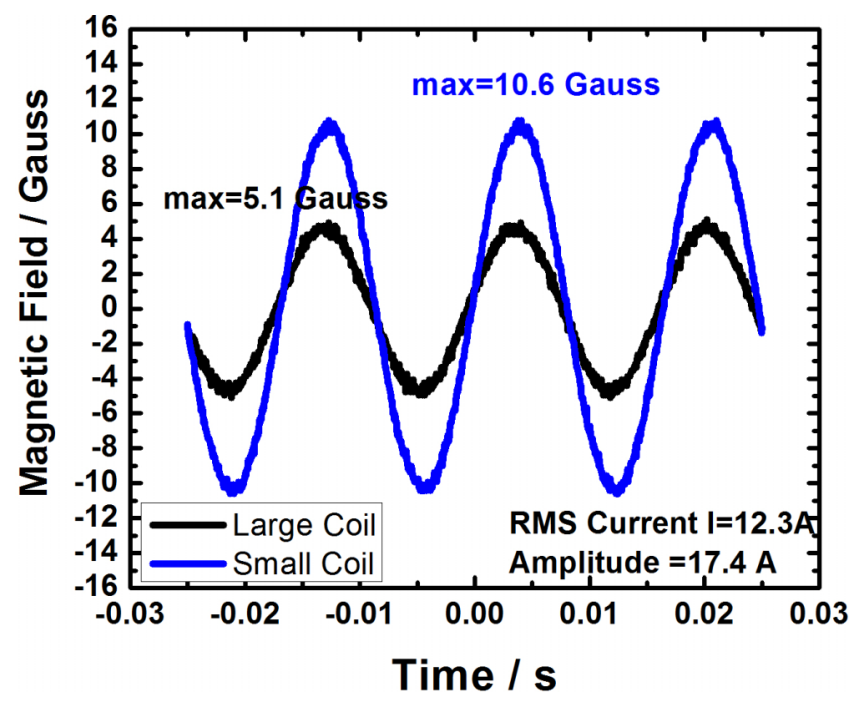

FIG. 3. The graph shows the measurement of the magnetic field of the nested Helmholtz coils from low frequency alternating current from the electrical outlet. or borosilicate glass sample. The exiting laser beam passes through an analyzer (calcite prism) oriented $45^{\circ}$ relative to the orientation of the linear polarized light, which allows splitting the laser into two equal intensity beams that are projected on a balanced photodiode (Model 2307 Large-Area AdjustableGain Balanced Photoreceivers from Newport, Inc.). The photoreceiver is placed far from the magnetic field to minimize any electronic interference from magnetic fields directly influencing signal on the photoreceivers. When the magnetic field is present, the rotation of magnetic field appears as positive or negative signal (depending on the direction of the magnetic field) on the oscilloscope from the balanced photodiodes. Based on the characteristics of the photoreciever and the optical power difference, the magnitude of the Faraday rotation signal is calculated. By comparing the measured signal with the value from the Faraday equation of the material, the magnetic field is calculated. Figure 4 demonstrates that the signal from the Rogowski sensor and the optical measurement are linearly proportional. Therefore, both measurements can be used to evaluate/calculate the magnetic fields of the coils. In the experiments below, the signals from the Rogowski coils are used as an indicator of the magnetic field strength.

Figure 5(a) shows the effect of changing the delay between the magnetic pulses on the two different coils, which results in different shaped magnetic fields. Changing the timing, the coils can produce linear alternating magnetic fields or rotating magnetic fields. Figure 4(b) shows the direct output from the Rogowski coil pairs fixed at a given time delay. This signal is integrated to obtain the current signal as described earlier in Figure 4. The direction of the rotating field can be also manipulated by changing the initial direction of the current in the coils resulting in left or right rotating magnetic fields. The maximum discharge of the capacitors used in the experiment is $10000 \mathrm{~V}$, which corresponds to $2850 \mathrm{~J}$ of energy. In a single shot, the maximum calculated temperature increase of the coil from this energy is $1.6{ }^{\circ} \mathrm{C}$ based on the weight of the copper coils and its heat capacity, but in reality it is probably much less than that since this simple estimate does not take the weight of the cables and the capacitor into account. The measured rotating magnetic field amplitude is $200 \mathrm{mT}$ at full discharge (10 $000 \mathrm{~V}$ charge for the larger coil). This is about 2.5 times less magnetic field than what is calculated from 

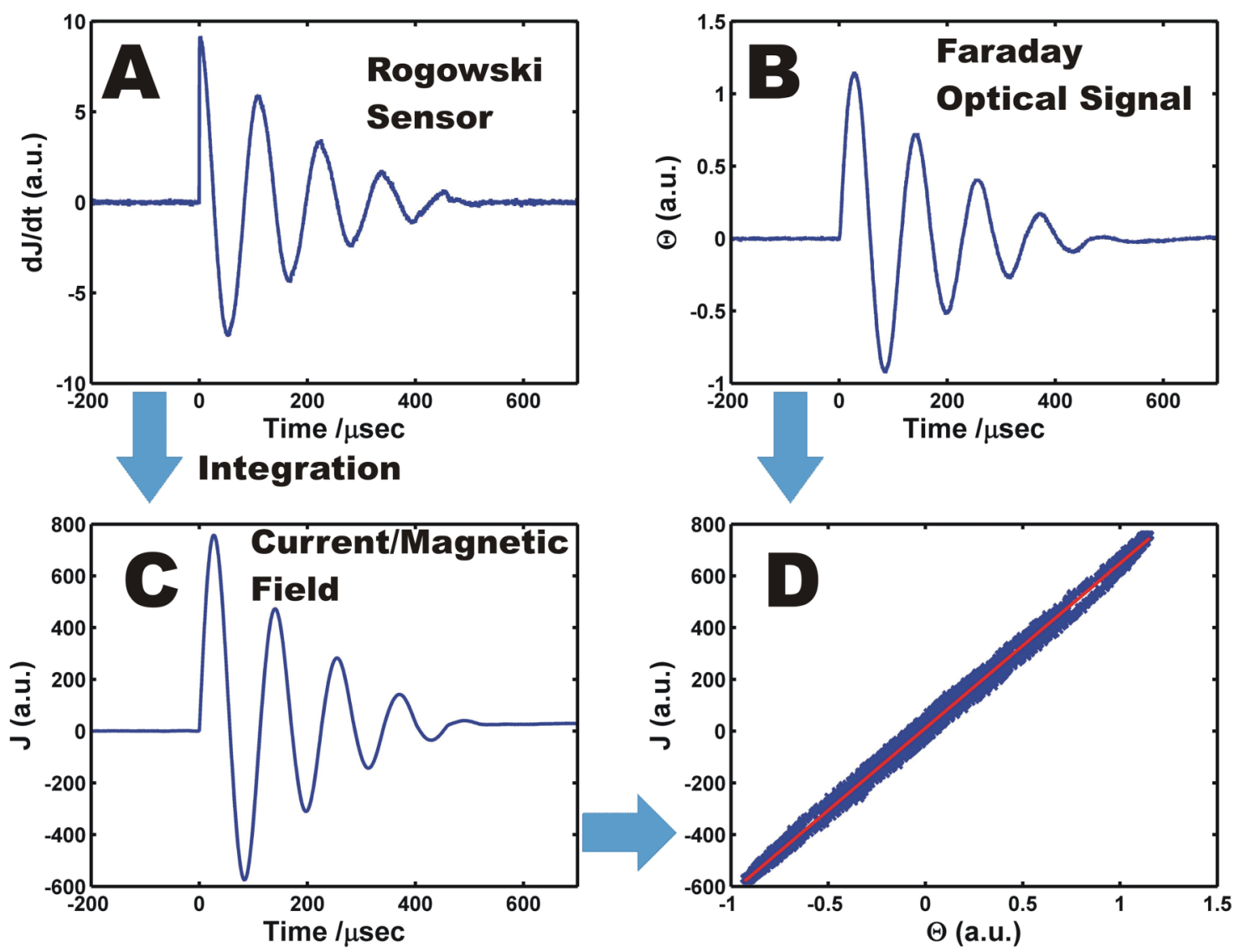

FIG. 4. Magnetic field of coil is measured via electronic sensor from the Rogowski coil (a) and the measurement of the Faraday rotation (b) of water placed inside the coils. For this calibration procedure, only one of the coils is fired. The direction of magnetic field is parallel to the 632 nm laser used for the Faraday measurement. The integrated Rogowski signal (c) is linearly proportional to the signal from the optical measurement (d). After calibration, the signals from the two Rogoswki sensors allow monitoring the magnitude and direction magnetic fields inside the coils for the rotating magnetic fields.
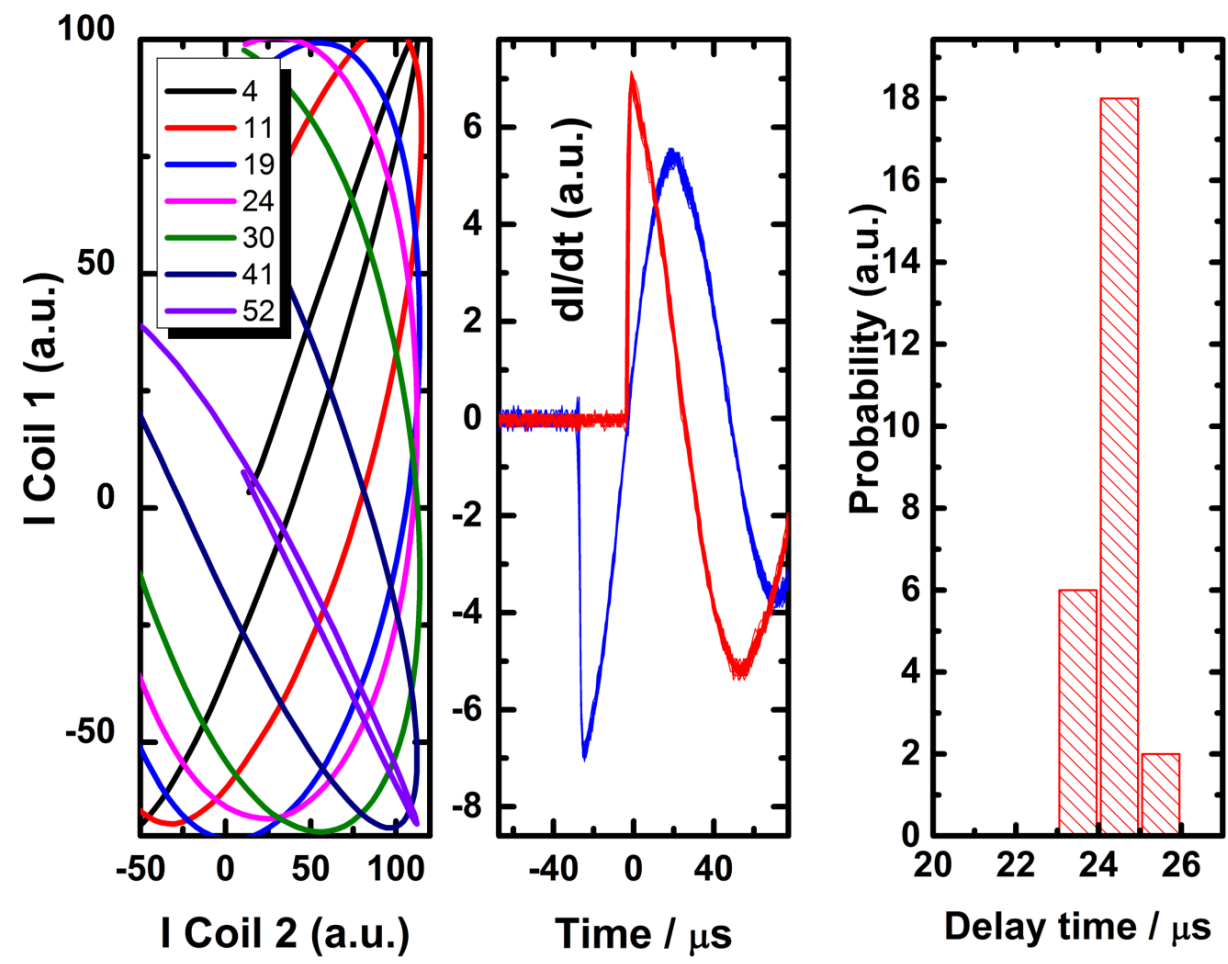

FIG. 5. LEFT: (a) Current signal from the two coils of the nested Helmholtz coils with various time delays. MIDDLE: (b) Direct signals from the Rogowski coils for the inner and outside Helmholtz coil from 26 simultaneous discharges. RIGHT: (c) Histogram of the timing jitter of the inner and outside coil firing from 26 shots. 

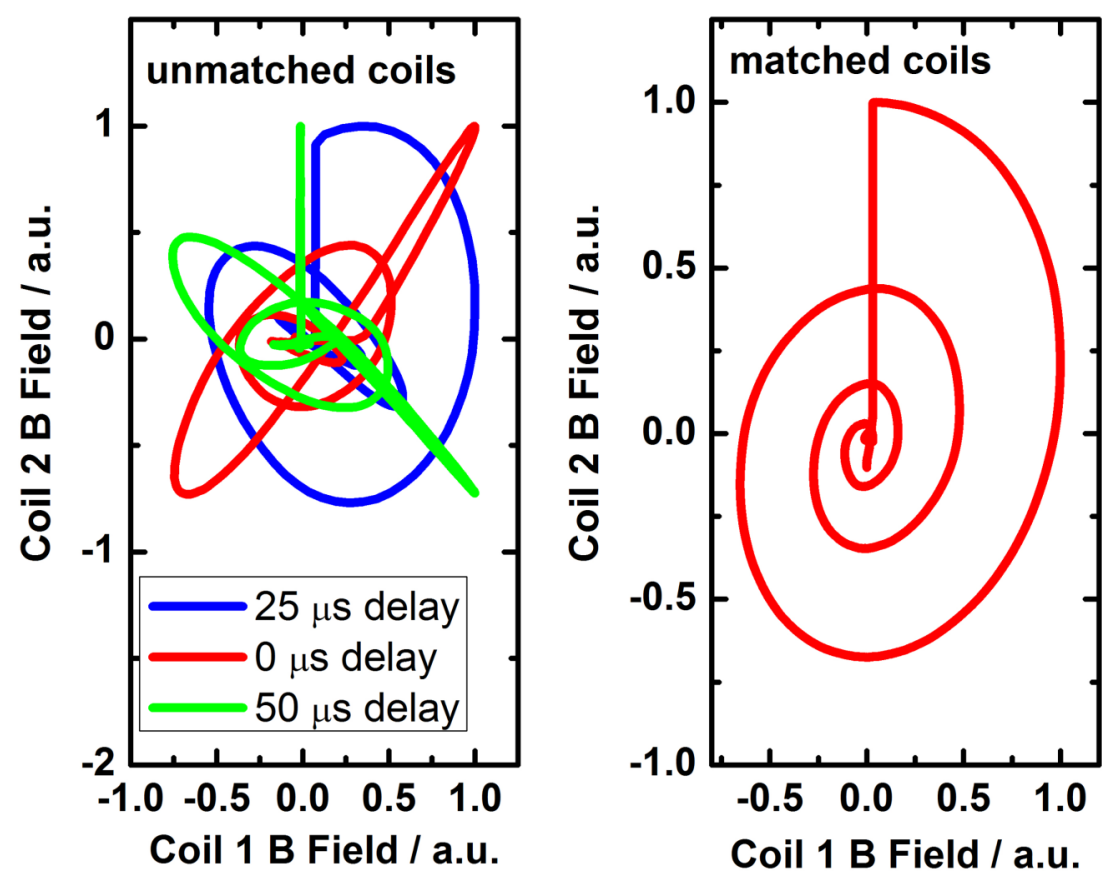

FIG. 6. Effect of lack of inductance matching in the two circuits, which results in resonance frequency mismatch. LEFT figure shows the unmatched Helmholtz coil where the rotating field can be kept only for a small period of the oscillation. RIGHT shows the matched coil which results in rotating magnetic field on the duration of the magnetic pulse.

modeling the discharge circuit and comparing the current with the data from Figure 3. We think that with careful optimization of the contact points in the coils, the current can be further increased, resulting in better agreement between the theory and practice. Timing of the magnetic pulses is important so that the desired rotating fields can be produced. The data on the time reproducibility of the magnetic pulses indicate that the jitter of the triggering in this system is relatively low. The timing jitter of the relative trigger remains under $1 \mu \mathrm{s},{ }^{18}$ which allows accurate timing to produce rotating fields for the few tens of kilohertz underdamped magnetic pulses.

Finally, it is important to match the inductance of the coils to produce pulses where the current and magnetic pulses remain in phase for the entire duration of the rotating magnetic field. This is demonstrated in Figure 6, which shows two separate cases. When the inductances of the coils are not matched, the various time delays will result in "scrambled" magnetic pulses. When the inductances of both coils are matched with the help of installing additional cable length to one of the coil circuits, the magnetic pulses become identical in both coils, which yield the desired rotating magnetic pulses. The magnitude of the rotating magnetic field is calculated from the current measurements from the Rogowski coils during the high voltage discharge.

In order to test the homogeneity of the rotating magnetic field, calculation is performed by using finite element method (FEM) implemented with COMSOL Multiphysics (Burlington, MA). This 3D model includes two pairs of coils, with the larger coil centered on the y-axis, and the smaller coil centered on the $\mathrm{x}$-axis. The model specifies a current density on each coil (out of phase) and solves for the magnetic vector potential and field strength at $100 \mathrm{kHz}$. Figure 7 illustrates the homogeneity of the computed magnetic fields within the region enclosed by the coils. The modeled region is discretized
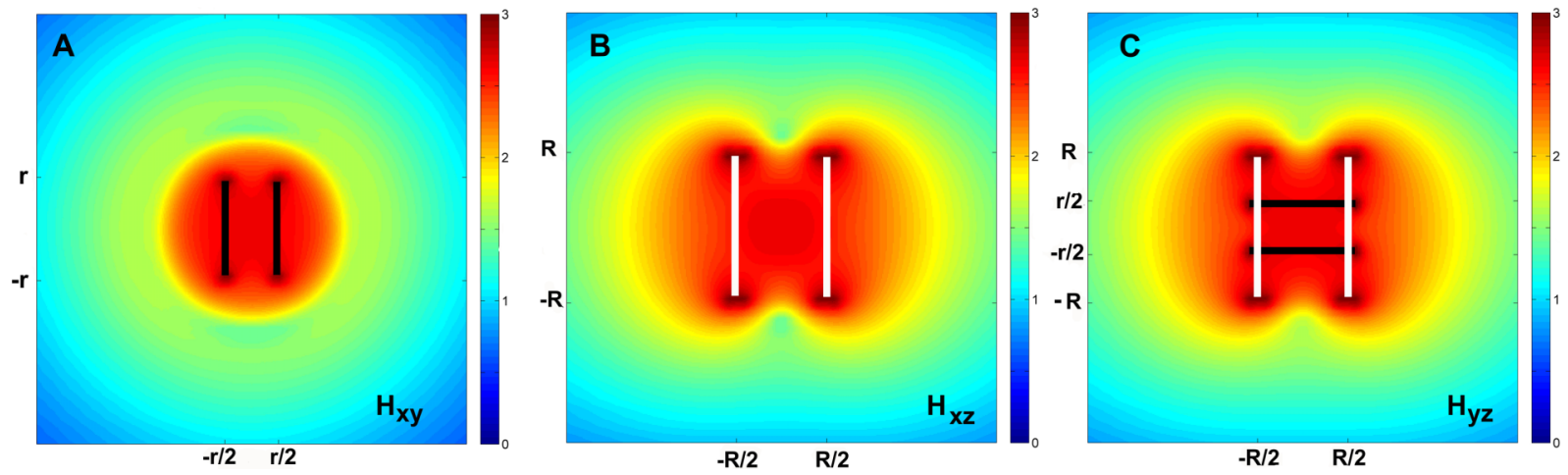

FIG. 7. Calculated rotating magnetic field amplitude $(\log (\mathrm{H}))$ for a $100 \mathrm{kHz}$ continuous operation of the in different planes for the nested Helmholtz used in the experiment. The dimensions of the coils are shown parametrically, where $\mathrm{r}$ is the radius of the smaller inner coil and $\mathrm{R}$ is the radius of the larger outer coil. The calculation shows that the rotating magnetic field is uniform in all directions in the center of the nested Helmholtz coils. 
with an inhomogeneous tetrahedral mesh, including 81398 elements. A frequency domain iterative solver (geometric multigrid) is used to compute the magnetic field strength at all points in the simulation domain. The variation of the magnetic field amplitude inside the inner coil is few percent relative to the absolute magnitude of the magnetic field. The calculation presented above is shown for the idealized 2D Helmholtz coil. The dimension of experimental coils used here shows a few percent deviation from the idealized Helmholtz coil; with careful adjustment of the manufacturing process, the coil can very closely approximate the idealized 2D Helmholtz coil.

\section{CONCLUSIONS}

In this article, the construction and operation of a novel Helmholtz are demonstrated to generate strong rotating magnetic field. The design shows scalability by manipulating several factors: increasing the number of plates used to construct the coils, reducing its dimensions, and increasing discharge voltage. The nested Helmholtz coil design could also be used in magnetic hypothermia experiments where minimizing the resistive heating is important at high frequencies due to the skin effects of the current at several tens of kilohertz.

\section{ACKNOWLEDGMENTS}

The authors would like to acknowledge the NSF (Grant Nos. NSF-1157044 and NSF-1128570 to VC) and the Terry Johnson Cancer Center for funding. They would like to acknowledge also Harvey McCarter for privately donating funds for this project and Ron Jackson at the Department of Chemistry, Kansas State University in the assembly and construction of the electromagnet.

\footnotetext{
${ }^{1}$ A. Jordan, R. Scholz, P. Wust, H. Fahling, and R. Felix, "Magnetic fluid hyperthermia (MFH): Cancer treatment with AC magnetic field induced excitation of biocompatible superparamagnetic nanoparticles," J. Magn. Magn. Mater. 201, 413-419 (1999).

${ }^{2}$ W. Wernsdorfer, E. B. Orozco, K. Hasselbach, A. Benoit, B. Barbara, N. Demoncy, A. Loiseau, H. Pascard, and D. Mailly, "Experimental evidence
}

of the Néel-Brown model of magnetization reversal,” Phys. Rev. Lett. 78, 1791-1794 (1997).

${ }^{3}$ S. Mornet, S. Vasseur, F. Grasset, and E. Duguet, "Magnetic nanoparticle design for medical diagnosis and therapy," J. Mater. Chem. 14, 2161-2175 (2004).

${ }^{4}$ E. Duguet, S. Vasseur, S. Mornet, and J. M. Devoisselle, "Magnetic nanoparticles and their applications in medicine," Nanomedicine 1, 157-168 (2006).

5 J. H. Lee, J. T. Jang, J. S. Choi, S. H. Moon, S. H. Noh, J. W. Kim, J. G. Kim, I. S. Kim, K. I. Park, and J. Cheon, "Exchange-coupled magnetic nanoparticles for efficient heat induction," Nat. Nanotechnol. 6, 418-422 (2011).

${ }^{6}$ V. A. Sharapova, M. A. Uimin, A. A. Mysik, and A. E. Ermakov, "Heat release in magnetic nanoparticles in AC magnetic Fields," Phys. Met. Metallogr. 110, 5-12 (2010).

${ }^{7}$ Y. L. Raikher and V. I. Stepanov, "Energy absorption by a magnetic nanoparticle suspension in a rotating field," J. Exp. Theor. Phys. 112, 173-177 (2011).

${ }^{8}$ G. Podaru, S. Ogden, A. Baxter, T. Shrestha, S. Q. Ren, P. Thapa, R. K. Dani, H. W. Wang, M. T. Basel, P. Prakash et al., "Pulsed magnetic field induced fast drug release from magneto liposomes via ultrasound generation," J. Phys. Chem. B 118, 11715-11722 (2014).

${ }^{9}$ H. W. Wang, T. B. Shrestha, M. T. Basel, R. K. Dani, G. M. Seo, S. Balivada, M. M. Pyle, H. Prock, O. B. Koper, and P. S. Thapa, "Magnetic$\mathrm{Fe} / \mathrm{Fe}_{3} \mathrm{O}_{4}$-nanoparticle-bound $\mathrm{SN} 38$ as carboxylesterase-cleavable prodrug for the delivery to tumors within monocytes/macrophages," Beilstein J. Nanotechnol. 3, 444-455 (2012).

${ }^{10}$ M. T. Basel, S. Balivada, H. W. Wang, T. B. Shrestha, G. M. Seo, M. Pyle, G. Abayaweera, R. Dani, O. B. Koper, and M. Tamura, "Cell-delivered magnetic nanoparticles caused hyperthermia-mediated increased survival in a murine pancreatic cancer model," Int. J. Nanomed. 7, 297-306 (2012).

${ }^{11}$ R. S. Rachakatla, S. Balivada, G. M. Seo, C. B. Myers, H. W. Wang, T. N. Samarakoon, R. Dani, M. Pyle, F. O. Kroh, and B. Walker, "Attenuation of mouse melanoma by A/C magnetic field after delivery of bi-magnetic nanoparticles by neural progenitor cells," ACS Nano 4, 7093-7104 (2010).

${ }^{12}$ C. Rinaldi, F. Gutman, X. W. He., A. D. Rosenthal, and M. Zahn, "Torque measurements on ferrofluid cylinders in rotating magnetic fields," J. Magn. Magn. Mater. 289, 307-310 (2005).

${ }^{13}$ M. D. Tomasini, C. Rinaldi, and M. S. Tomassone, "Molecular dynamics simulations of rupture in lipid bilayers," Exp. Biol. Med. 235, 181-188 (2010).

${ }^{14} \mathrm{~S}$. Suresh, "Biomechanics and biophysics of cancer cells," Acta Biomater. 3, 413-438 (2007).

${ }^{15}$ F. Bitter, "New developments in high-magnetic-field research," Phys. Today 14(9), 22-28 (1961).

${ }^{16}$ K. Mackay, M. Bonfim, D. Givord, and A. Fontaine, "50 T pulsed magnetic fields in microcoils," J. Appl. Phys. 87, 1996-2002 (2000).

${ }^{17}$ C. E. Waring and R. L. Custer, "Absolute Verdet constants for water over a range of temperatures and visible wave lengths," J. Am. Chem. Soc. 74, 2506-2509 (1952).

${ }^{18}$ L. L. Small, D. C. D. McKen, and A. A. Offenberger, "Low-jitter, low-inductance, electrically triggered spark gap," Rev. Sci. Instrum. 55, 1084-1089 (1984). 